\title{
Preparation and Characterization of Hydroxyapatite-Coated Selenium Nanoparticles and their Interaction with Osteosarcoma (SaOS-2) Cells
}

\author{
Thiagarajan Hemalatha - Gunasekaran Krithiga • Baskar Santhosh Kumar • Thotapalli P. Sastry
}

Received: 21 February 2014/Revised: 2 June 2014/Published online: 28 September 2014

(C) The Chinese Society for Metals and Springer-Verlag Berlin Heidelberg 2014

\begin{abstract}
Nanotechnology has taken a firm step to revolutionize the field of orthopedic implants. Current research on bone implants focuses to develop implants with multifaceted functions viz., osteoinduction, chemoprevention, antimicrobial action etc., especially for cancerous bone resection. The objective of the present study was to synthesize a novel composite for bone implants, possessing the above properties. Selenium was selected owing to its chemopreventive and chemotherapeutic properties. Hydroxyapatite was selected owing to its bioactivity and similarity in composition to bone mineral properties. Selenium nanoparticles were prepared by chemical reduction method and coated with hydroxyapatite. Hydroxyapatite-coated selenium nanoparticle (HASnp) was characterized physico-chemically using fourier transform infrared spectroscopy, X-ray diffractometry, scanning electron microscope, and energy-dispersive X-ray spectroscopy. HASnp was analysed in vitro using SaOS-2 cell line. Enhanced cell proliferation and alkaline phosphatase activity were observed in HASnp-treated cells. The results indicate that HASnp is highly suitable for the use in orthopedic applications.
\end{abstract}

\section{KEY WORDS: Osteogenesis; Biocompatibility; Bone tissue engineering; Biomaterials; Biomedical} applications

\section{Introduction}

Biomaterials used for the preparation of orthopedic implants are now benefitted by the advances in the field of nanotechnology. Metals and polymers are the most commonly used materials for bone implants. Metallic bone implants possess numerous problems limiting their longterm efficacy, such as poor prolonged osseointegration, stress shielding, and corrosion under in vivo environments [1]. Also, during bone malignancies such as osteosarcoma, bone resection is employed as a better control, which

Available online at http://link.springer.com/journal/40195

T. Hemalatha - G. Krithiga · B. Santhosh Kumar .

T. P. Sastry $(\bowtie)$

Bioproducts laboratory, Central Leather Research Institute, Chennai 600 020, Tamilnadu, India

e-mail: sastrytp@hotmail.com necessitates the need for reconstruction often with a orthopedic implant [2]. Hence there is a need for the development of orthopedic materials possessing multifaceted functions viz., enhanced osteoblast proliferation, antitumor activity, anti-bacterial activity etc., which could serve the purpose of healthy bone growth, while inhibiting cancer reoccurrence and/or infections. Since cancer cells impair the functions of osteoblasts and induce osteoblast apoptosis [3], it is important to incorporate materials with chemopreventive action in bone implants used after cancer resection.

Selenium, well known for its chemopreventive and chemotherapeutic action [4-6], is an essential trace element that has multiple beneficial effects for human health. It regulates at least 25 selenoproteins, which contains the twenty first genetically encoded protein amino acid selenocysteine [7]. There is ample evidence to prove that selenium has anti-cancer [8] and anti-bacterial properties [9]. A number of mechanisms have been postulated to 
explain the chemopreventive action of selenium which mainly includes reduction in DNA damage and oxidative stress [10]. A recent report [11] suggests that intraperitoneal administration of selenium nanoparticles is a safe and effective means of preventing growth of cancer cells in peritoneal cavity. Selenium nanoparticles are most effective as smaller particles [12], and they act as potent antioxidants with reduced risk of toxicity [13]. Nanostructured selenium was reported to enhance bone cell adhesion [1]. Increased osteoblast functions viz., adhesion, proliferation etc., were observed on selenium nanoclusters-coated titanium substrates [6]. Li et al. [14] reported that functionalized selenium nanoparticles possess nephroprotective activity.

Hydroxyapatite, is also used as a bone graft material in orthopedic implants since it possesses exceptional biocompatibility and bioactivity properties with respect to bone cells and tissues, probably due to its similarity with the hard tissues of the body. Nanosized hydroxyapatite is the main component of mineral bone [15]. Since, hydroxyapatite was found to be capable of supporting bone ingrowth and osseointegration [16], it had significant success in strengthening the fixation between the implant and bone tissue, thus reducing the healing period during orthopedic implantation practice [17]. Hydroxyapatite nanoparticles possess better properties viz., surface grain size, pore size, wettability, etc. compared to conventional ceramic implants, which aids in enhanced osteoblast adhesion and long-term functionality [15].

The main objective of this study was to investigate the potential use of selenium nanoparticles in bone implants. Nanoparticles, because it is the length scale that most of the body's natural materials possess [1]. Several methods including gamma irradiation and laser ablation had been applied to synthesize selenium nanoparticles but chemical reduction is the most widely used method [13]. In this study, selenium nanoparticles were prepared by chemical reduction and coated with hydroxyapatite. Physicochemical characterization of hydroxyapatite-coated selenium nanoparticles (HASnp) was done and its osteoinductive effects were evaluated in vitro (Graphical abstract).

\section{Materials and Methods}

\subsection{Preparation of Selenium Nanoparticles}

Selenium nanoparticles were prepared using solution-phase approach by reducing selenious acid solution with ascorbic acid in the presence of chitosan [18].
2.2 Preparation of Hydroxyapatite-Coated Selenium Nanoparticles

$57.5 \mathrm{mg}$ of $\mathrm{Ca}(\mathrm{OH})_{2}$ was mixed with $0.5 \mathrm{~mL}$ of $4 \%$ ethanol. $50 \mu \mathrm{L}$ of chitosan-capped selenium nanoparticles was added to this solution and it was stirred well using magnetic stirrer at $80{ }^{\circ} \mathrm{C} .420 \mu \mathrm{L}$ of phosphoric acid was added to it and the $\mathrm{pH}$ value was adjusted to 10 . After precipitation, the supernatant was discarded and the pellet was separated. The hydroxyapatite-coated selenium nanoparticles (HASnp) obtained was dried well.

\subsection{Characterisation}

\subsubsection{UV-visible Spectroscopy}

UV-visible spectra of the nanodispersions (selenium nanoparticles) were recorded in Jasco V 30 spectrophotometer with matched pair of quartz cell. Distilled water was used as blank.

\subsubsection{Fourier Transform Infrared Spectroscopy (FTIR)}

Chitosan, chitosan-capped selenium nanoparticles, and HASnp were analysed by FTIR (Nicolet Magna IR 560, USA) in the range of $500-4,000 \mathrm{~cm}^{-1}$ with resolution 4 and 128 times scanning.

\subsubsection{Scanning Electron Microscope (SEM)}

Samples were coated with ultra thin layer of gold using an ion coater (fisons sputter coater) under the following conditions viz., $13.33 \mathrm{~Pa}$ pressure, $0.2 \mathrm{~A}$ current, and $70 \mathrm{~s}$ coating time. Surface structure was observed by scanning electron microscope (SEM model-LEICA stereoscan 440) using $15 \mathrm{kV}$ accelerating voltage.

\subsubsection{Energy-Dispersive X-ray Spectroscopy (EDX)}

For EDX analysis, samples were mounted on holders and $6 \mathrm{~nm}$ sputter coated with carbon by means of a MED 020 sputter device (Bal-Tec). In a Philips scanning electron microscope (Philips, Eindhoven, the Netherlands), EDX analyses were performed at 5 and $10 \mathrm{kV}$.

\subsubsection{Transmission Electron Microscope (TEM)}

The selenium nanoparticles were characterized using Teenal 10 Philips transmission electron microscope. Samples for TEM studies were prepared by placing a drop of nanodispersion on a carbon-coated $\mathrm{Cu}$ grid, and solvent was evaporated at room temperature. 


\subsubsection{X-ray Diffractometry (XRD)}

HASnp samples were analysed on a Siemens 500 X-ray powder diffractometer in a flat plane geometry using a source of $\mathrm{Cu} K_{\alpha}$ with the wavelength of $0.15406 \mathrm{~nm}$. The patterns were recorded in the $2 \theta$ region of $10^{\circ}-90^{\circ}$ in steps of $0.001^{\circ}$ with a counting time of $1 \mathrm{~s}$ in each step.

\subsection{Cell Culture Studies}

In vitro assay was performed on human osteosarcoma cell line (SaOS-2) obtained from National Centre for Cell Science, Pune, India. The cells were grown on McCoy's 5A medium (HiMedia) supplemented with $15 \%$ fetal bovine serum (Gibco Laboratories) and antibiotics (streptomycin, penicillin, and amphotericin B, [Sigma]) and incubated under standard conditions $\left(37^{\circ} \mathrm{C}\right.$, humidified, $5 \% \mathrm{CO}_{2}$ and $95 \%$ air).

\subsubsection{MTT Assay}

Biocompatibility was assessed using MTT [3-(4,5Dimethylthiazol-2-yl)-2,5-diphenyltetrazolium bromide] assay. SaOS-2 cells $\left(1 \times 10^{4}\right)$ grown on 96-well plate at a volume of $200 \mu \mathrm{L}$ per well was exposed to various concentrations $(25,50,100,150$, and $200 \mu \mathrm{g} / \mathrm{mL})$ of HASnp. The cell viability was measured after $48 \mathrm{~h}$ using MTT assay [19]. MTT was added at the end of incubation period, and the plates were incubated for $4 \mathrm{~h}$ at $37{ }^{\circ} \mathrm{C}$. Following incubation, media were aspirated completely and MTT formazan crystals formed were dissolved by the addition of dimethyl sulfoxide, and the absorbance was read at $570 \mathrm{~nm}$ using a multiwell plate reader (Multiskan Ascent VI.24). Untreated wells served as cell control. Data were normalized to control to evaluate the effect of HASnp on cells.

\subsubsection{Alkaline Phosphatase Activity}

Alkaline phosphatase (ALP) activity was measured in SaOS2 cells grown in McCoy's 5A medium (HiMedia) supplemented with $15 \%$ fetal bovine serum (Gibco Laboratories), antibiotics (streptomycin, penicillin, and amphotericin B, [Sigma]), $\beta$-glycerophosphate (100 mmol/L), ascorbic acid $(100 \mathrm{nmol} / \mathrm{L})$, and dexamethasone $(10 \mathrm{nmol} / \mathrm{L})$ (SigmaAldrich, Bangalore, India). Cells were seeded onto 24-well plates, and cultures were treated with various concentrations $(25,50,100,150$, and $200 \mu \mathrm{g} / \mathrm{mL})$ of HASnp and ALP activity was measured after different periods of incubation. Untreated wells served as control. After incubation, the cells were thoroughly washed with phosphate-buffered saline (PBS), and then lysed using ice-cold lysis buffer $(20 \mathrm{mmol} / \mathrm{L}$ Tris-HCl-pH7.5, $150 \mathrm{mmol} / \mathrm{L} \mathrm{NaCl}, 1 \% \mathrm{v} / \mathrm{v}$ Triton X-100), followed by centrifugation at $3300 \mathrm{~g}$, at $4{ }^{\circ} \mathrm{C}$ for $5 \mathrm{~min}$. The supernatant was carefully retrieved and stored at $4{ }^{\circ} \mathrm{C}$ until further use. Working solution of alkaline phosphatase (ALP) kit was prepared as per the manufacturer's guidelines. Lysis buffer was used as reference for absorbance. Then, absorbance was measured at $405 \mathrm{~nm}$ after $10 \mathrm{~min}$. The enzyme activity was calculated according to manufacturer's formula [20]. The experiment was done in triplicates.

\subsection{Statistical Analysis}

The results of the cell culture studies are given as mean \pm standard deviation of three individual experiments $(n=3)$. The comparison between means was done by oneway analysis of variance $(p<0.05)$ followed by Duncan's multiple range analysis using statistical software package SPSS, version 13.0.

\section{Results}

\subsection{Characterization}

\subsubsection{UV-Visible Spectrophotometer}

Ascorbic acid reduced colorless selenious acid to red-colored selenium nanoparticles, which had an absorbance maximum at $290 \mathrm{~nm}$ (Fig. 1). The size of the selenium nanoparticles could be in the range of $40 \mathrm{~nm}$.

\section{1 .2 FTIR}

The FTIR spectrum of chitosan and chitosan-capped selenium nanoparticles is given in Fig. 2a and b, respectively. The FTIR spectrum of chitosan shows a broad $\mathrm{OH}$ stretching absorption band between 3,412 and $2,921 \mathrm{~cm}^{-1}$ and the aliphatic $\mathrm{C}-\mathrm{H}$ stretching between 2,921 and $2,880 \mathrm{~cm}^{-1}$. Another major absorption band between 1,258

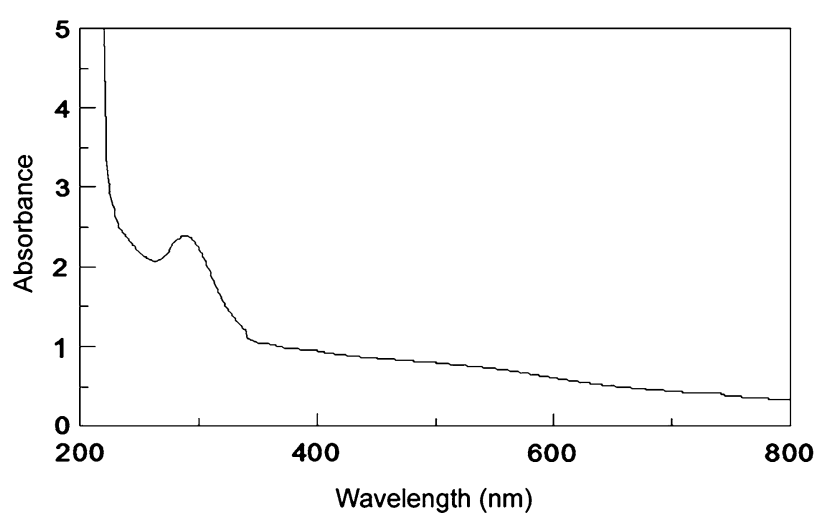

Fig. 1 UV-Vis spectrum of colloidal chitosan-capped selenium nanoparticles 

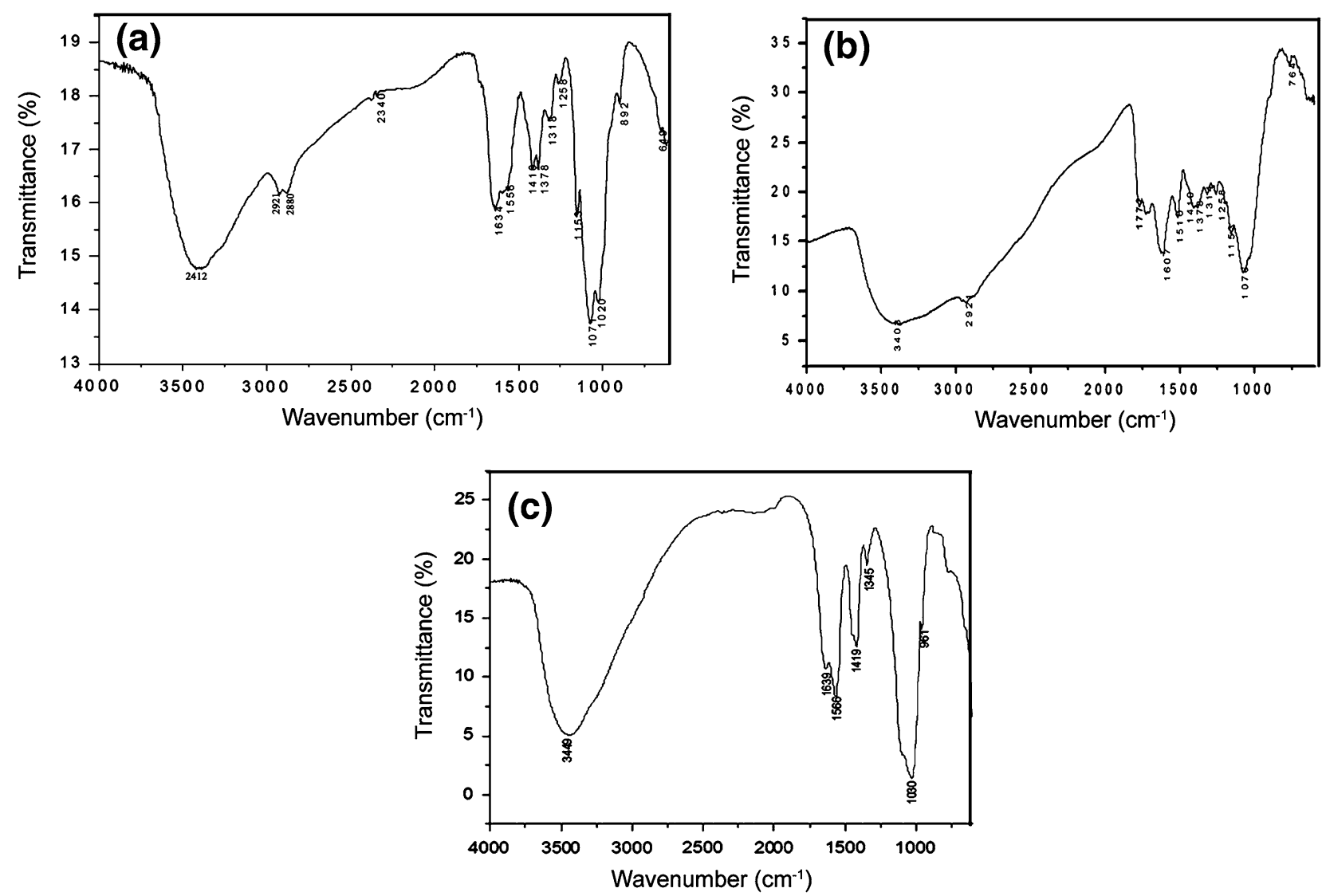

Fig. 2 FTIR spectra of chitosan a, chitosan-capped selenium nanoparticles $\mathbf{b}$, hydroxyapatite-coated selenium nanoparticles $\mathbf{c}$

and $1,020 \mathrm{~cm}^{-1}$ represents the free primary amino group $\left(-\mathrm{NH}_{2}\right)$ at $\mathrm{C}_{2}$ position of glucosamine, a major group present in chitosan. Peak at $1,634 \mathrm{~cm}^{-1}$ represents acetylated amino group of chitin. Peak at $1,378 \mathrm{~cm}^{-1}$ represents the $\mathrm{C}-\mathrm{O}$ stretching of primary alcoholic group $\left(-\mathrm{CH}_{2}-\mathrm{OH}\right)$. The FTIR spectrum of chitosan-capped selenium nanoparticles shows a shift in $\mathrm{OH}$ stretching absorption band from $3,412 \mathrm{~cm}^{-1}$ of chitosan to $3,403 \mathrm{~cm}^{-1}$. Peak at $1,634 \mathrm{~cm}^{-1}$ of chitosan represents acetylated amino group of chitin, shifted to $1,607 \mathrm{~cm}^{-1}$. The FTIR spectrum of selenium composite is given in Fig. 2c. The peak at $961.1 \mathrm{~cm}^{-1}$ corresponds to the symmetric stretching made of phosphate. The peak at $1,032 \mathrm{~cm}^{-1}$ is assigned to a symmetric vibration of phosphate groups. This result indicates the formation of hydroxyapatite in the composite.

\subsubsection{SEM}

Scanning electron micrographs revealed the surface morphology of the materials, their shape, and size. The shape of the selenium nanoparticles was found to be spherical (Fig. 3a), and their size were found to be in the range
23-48 nm. SEM pictures of HASnp are given in Fig. 3b. The size of HASnp was in the range of $50-66 \mathrm{~nm}$. This increase in size may be due to the coating of hydroxyapatite on the selenium nanoparticles.

\subsubsection{EDX}

The EDX results (Fig. 4a) of selenium nanoparticles indicate the presence of selenium (about 68\%) in the material prepared, while the EDX of the composite HASnp (Fig. 4b) reveals the presence of calcium and phosphorous along with selenium. This result clearly indicates the coating of hydroxyapatite onto the selenium nanoparticles.

\subsubsection{TEM}

The TEM image of selenium nanoparticles is shown in Fig. 5. The size of the selenium nanoparticles was found to be around $23-46 \mathrm{~nm}$ which was comparable with the results of SEM. Some of the particles agglomerated and formed as a cluster. These clusters or aggregates were in the size range of $92 \mathrm{~nm}$. 

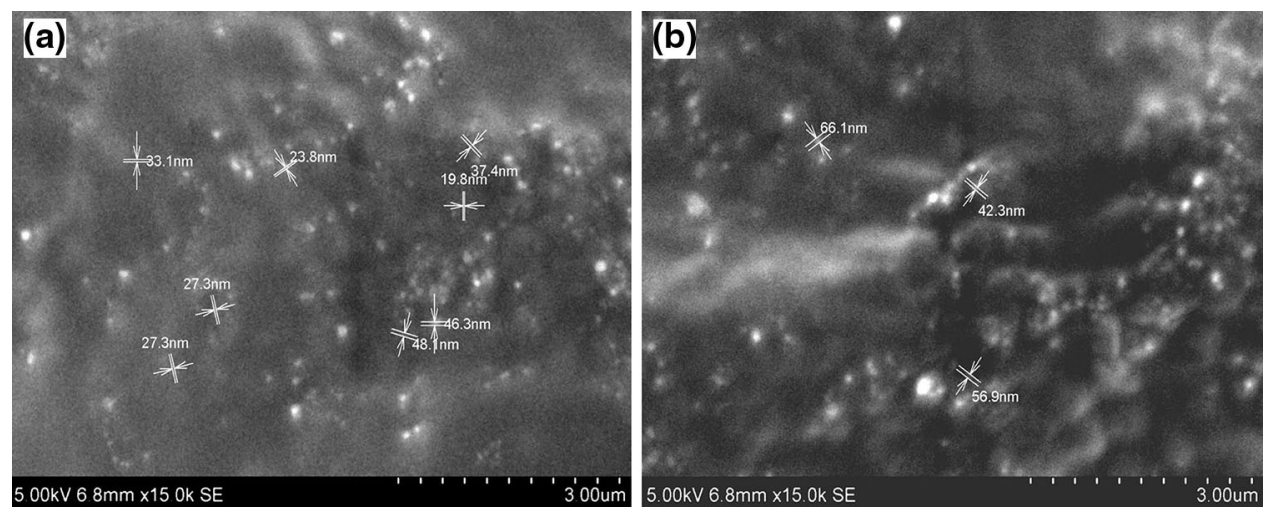

Fig. 3 SEM images of chitosan-capped selenium nanoparticles a and hydroxyapatite-coated selenium nanoparticles $\mathbf{b}$
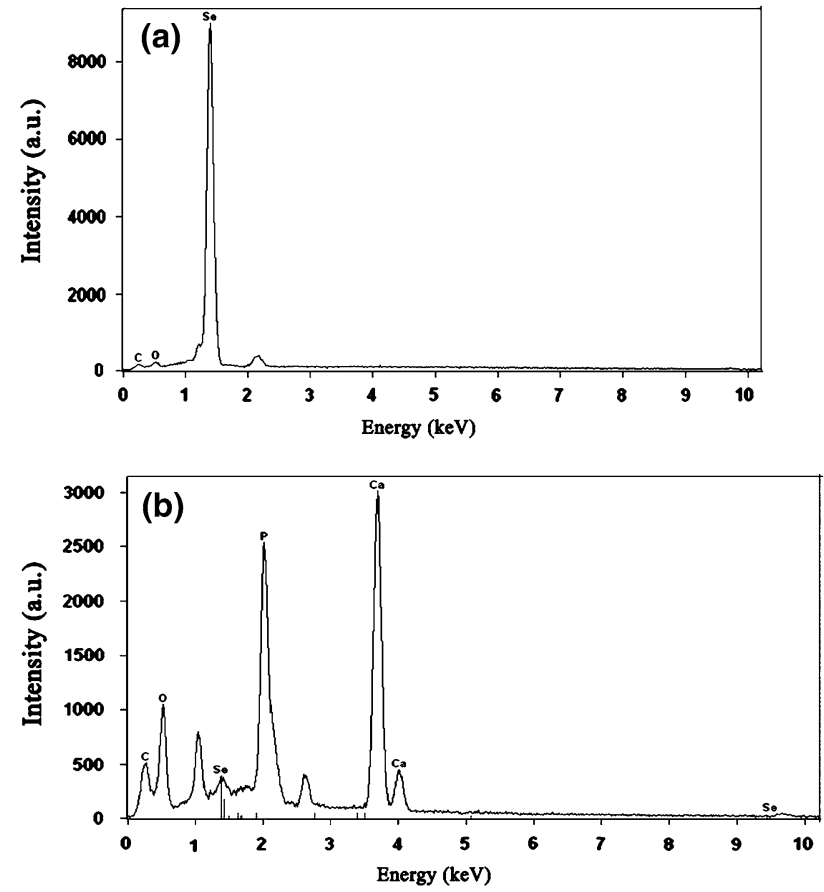

Fig. 4 EDX spectra of chitosan-capped selenium nanoparticles a and hydroxyapatite-coated selenium nanoparticles $\mathbf{b}$

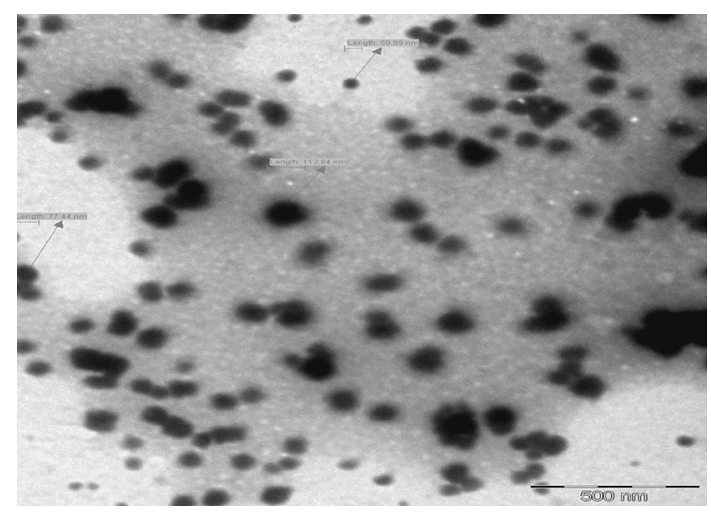

Fig. 5 TEM image of colloidal selenium nanoparticles

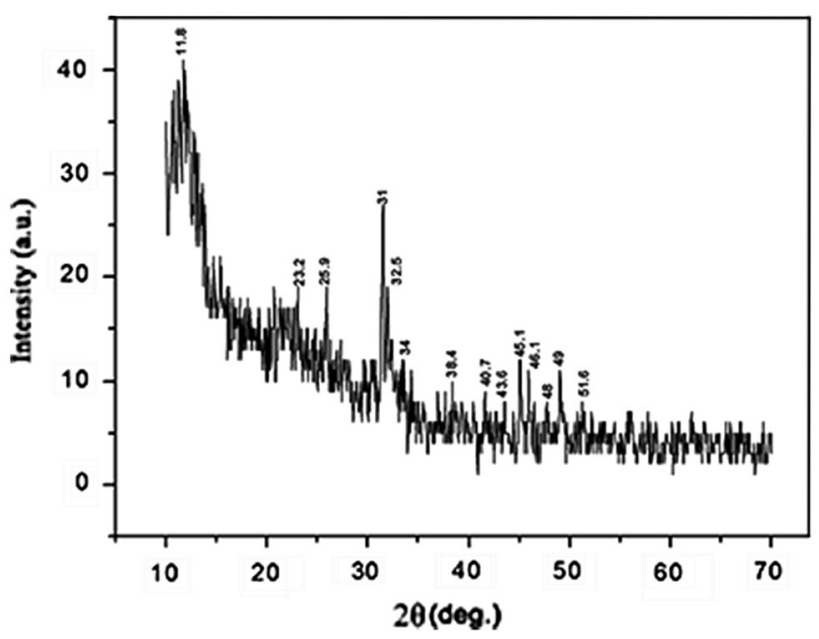

Fig. 6 X-ray diffraction pattern of hydroxyapatite-coated selenium nanoparticles

\subsubsection{XRD}

The XRD pattern of HASnp (Fig. 6) shows the characteristic diffraction peaks corresponding hydroxyapatite at $2 \theta$ position. The peaks at $31,32.5$ represent the crystalline hydroxyapatite form. There is a slight deviation in the peaks compared to original diffraction pattern which may be due to the selenium nanoparticles. The characteristic $\mathrm{X}$-ray diffraction peaks of selenium nanoparticles at 23.2, 45.1 , and 43.6 are seen in the XRD pattern of selenium hydroxyapatite. These results indicate the formation of hydroxyapatite on selenium nanoparticles.

\subsection{Cell Culture Studies}

\subsubsection{MTT Assay}

MTT assay revealed the biocompatible nature of HASnp. HASnp-treated groups exhibited significant $(p<0.05)$ 

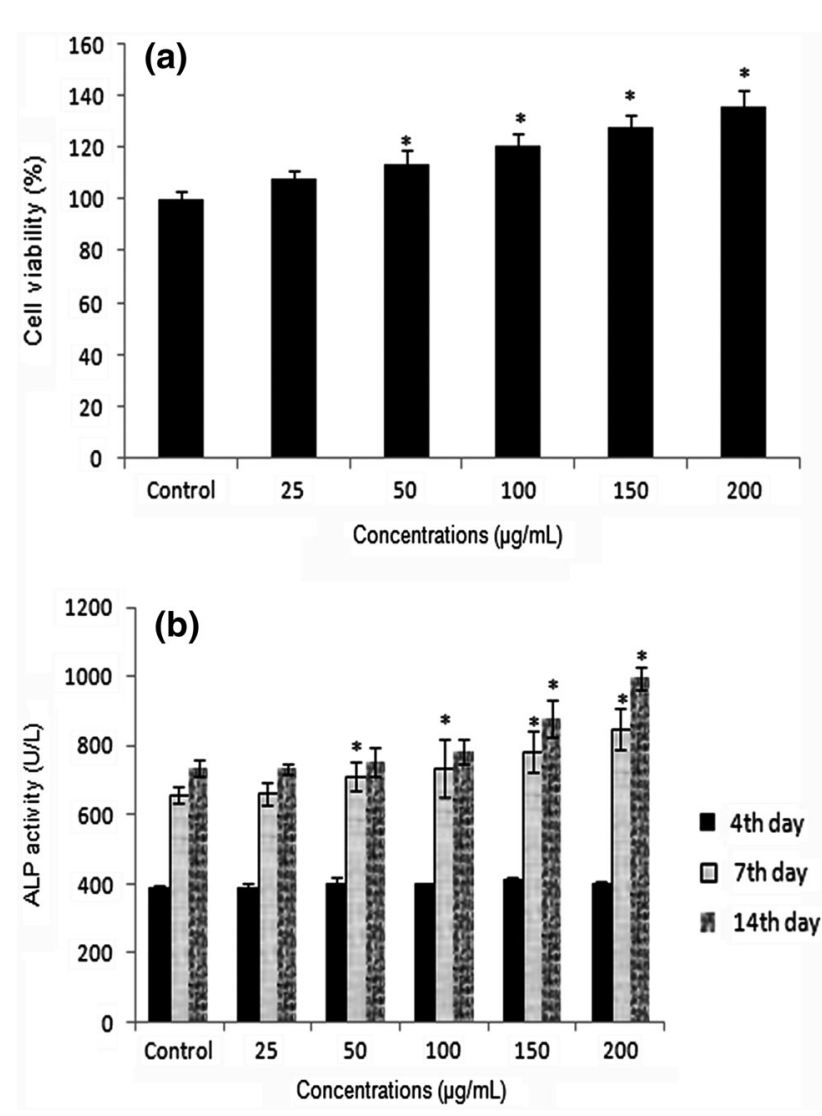

Fig. 7 a MTT assay (data are presented as mean \pm SD of three individual experiments $(n=3)$. ${ }^{*} p \leq 0.05$ as compared to control group, using Duncan's multiple range analysis); b Alkaline phosphatase activity (data are presented as mean \pm SD of three individual experiments $(n=3)$. ${ }^{*} p \leq 0.05$ as compared to control group, using Duncan's multiple range analysis)

increase in viability compared to control at all concentrations, except at $25 \mu \mathrm{g} / \mathrm{mL}$. Concentration-dependent increase in cell viability was observed (Fig. 7a).

\subsubsection{ALP Activity}

There was no significant difference in ALP activity among the groups on fourth day. Increased ALP activity was evident above $25 \mu \mathrm{g} / \mathrm{mL}$ concentration of HASnp on seventh day and at 150 and $200 \mu \mathrm{g} / \mathrm{mL}$ concentrations on fourteenth day, compared to control (Fig. 7b).

\section{Discussion}

Selenium nanoparticles in the recent past have been envisaged to have immense medical (free radical scavenging, anti-cancer, antioxidant, and anti-bacterial) applications and have emerged as a novel selenium source with the obvious advantage of reduced risk of selenium toxicity [21]. Hydroxyapatite is particularly interesting for implant purposes for its close similarities with mineral constituents of bone and tooth. Both in vivo and in vitro studies have proved hydroxyapatite to be a promising candidate in orthopedics [17]. Paraspour et al. [22] have reported that the coating of the metallic implants with hydroxyapatite would increase its biocompatibility and osseointegration. In the synthesis of selenium nanoparticles, chitosan solution is used as stabilizing agent. Polysaccharides are used as modifiers/stabilizers in the preparation of selenium particles in order to obtain significant materials for medical or food uses. The amino group of chitosan reacts with selenious acid and reduces elemental selenium in situ by ascorbic acid. Chitosan, a natural polysaccharide with potent biological properties [23] is used to prepare selenium nanoparticles envisioning its use in medical applications [18]. Kong et al. [24] reported that selenium nanoparticles prepared using gum arabic as a stabilizer possessed antioxidant properties. In UV-visible spectrophotometer, the spectra had maxima at $290 \mathrm{~nm}$. The $\lambda \max$ indicates that the majority of the nanoparticles are around 40-60 $\mathrm{nm}$. These results are in line with earlier studies [25].

SEM and TEM images showed spherical nanoparticles are around $40-60 \mathrm{~nm}$ in size which is in accordance with UV-visible spectrophotometer results. As the size of nanoparticles decreases, its activity increases. In previous studies, selenium nanoparticles were in the range of 40-190 nm [25]. Particle size is one of the most important parameters determining biocompatibilities and bioactivities of materials of therapeutic importance [26]. Selenium nanoparticles in the size of 5-200 $\mathrm{nm}$ directly scavenge free radicals in vitro in a size-dependent fashion [12]. The elemental composition analysis by TEM-EDX showed the presence of strong signals (68\%) of selenium, which may be due to the capping of selenium nanoparticles by chitosan.

The results of XRD and FTIR reveal the formation of hydroxyapatite on chitosan-capped selenium nanoparticles. SEM results clearly depict a difference in size between individual HASnp and chitosan-capped selenium nanoparticles. Increased size of HASnp could be due to hydroxyapatite formation. The results of EDX regarding the contents of $\mathrm{Ca}, \mathrm{P}, \mathrm{Se}$ in HASnp is in accordance with the previous FTIR and XRD results. Hence the physicochemical characterization has clearly proved the formation of HASnp composite.

SaOS- 2 cell line was selected for the study since it possesses several osteoblastic features and functions like osteoblast-like cells [27]. The biocompatibility of hydroxyapatite and selenium nanoparticles is already established. MTT study revealed that HASnp could significantly improve cell proliferation above $25 \mu \mathrm{g} / \mathrm{mL}$ in addition to its non-toxic nature. HA-coated magnetic 
nanoparticles were also reported to increase osteoblast densities [9]. It is understood from the study that HASnp is well tolerated by the cell and the enhanced proliferation of SaOS- 2 cells could be attributed to hydroxyapatite which serves as the main component of several bone substitutes already used in clinical applications [28].

ALP activity is a marker of bone formation and it plays a key role in bone mineralization by initiating and promoting the formation of hydroxyapatite in osteoblast matrix vesicles, which are then released into the extracellular matrix [9]. There was not much difference in ALP activity on fourth day, while HASnp-treated cells possessed enhanced ALP activity on seventh and fourteenth day, which conforms to the usual pattern of ALP expression by bone-like cells [20]. Similar results of increased ALP expression at $200 \mu \mathrm{g} / \mathrm{mL}$ at all time points have been reported for hydroxyapatite-coated iron oxide nanoparticles [9] and Fe-hydroxyapatite nanoparticles [28]. Hence, from the cell culture studies, it is clearly evident that HASnp could promote healthy bone cell functions viz., adhesion, proliferation etc., in addition to its already proven inhibitory effect on cancer cell functions.

\section{Conclusions}

HASnp was synthesized to incorporate the anti-cancer and anti-microbial properties of selenium nanoparticles in bone implants, along with healthy osteoblast functions. HASnp was characterized physico-chemically using FTIR, SEM, EDX, and XRD. Growth of SaOS-2 cells in the presence of HASnp revealed that it was biocompatible; it could enhance cell proliferation and ALP activity above $25 \mu \mathrm{g} / \mathrm{mL}$. HASnp combines the significant potentials of three bioactive components viz., selenium, chitosan, and hydroxyapatite and the results demonstrate its osteoinductive potential. Hence, it could be proposed for use in orthopedic applications, with further studies.

Acknowledgments The award of CSIR fellowship to T. Hemalatha and B. Santhosh Kumar is gratefully acknowledged.

\section{References}

[1] P. Tran, T.J. Webster, Int. J. Nanomed. 3, 391 (2008)

[2] Z. Shi, X. Huang, B. Liu, H. Tao, Y. Cai, R. Tang, J. Biomater. Appl. 25, 19 (2010)
[3] A.M. Mastro, C.V. Gay, D.R. Welch, H.J. Donahue, J. Jewell, R. Mercer, D. DiGirolamo, E.M. Chislock, K. Guttridge, J. Cell. Biochem. 91, 265 (2004)

[4] M. Navarro-Alarcon, M.C. Lopez-Martinez, Sci. Total Environ. 249, 347 (2000)

[5] W.Q. Wei, C.C. Abnet, Y.L. Qiao, S.M. Dawsy, Z.W. Dong, X.D. Sun, J.H. Fan, E.W. Gunter, P.R. Taylor, S.D. Mark, Am. J. Clin. Nutr. 79, 80 (2004)

[6] P.A. Tran, L. Sarin, R.H. Hurt, T.J. Webster, J. Biomed. Mater. Res. A 93, 1417 (2010)

[7] H. Steinbrenner, H. Sies, Biochim. Biophys. Acta 1790, 1478 (2009)

[8] M.I. Jackson, G.F. Combs Jr, Curr. Opin. Clin. Nutr. Metab. Care. 11, 718 (2008)

[9] P. Tran, T.J. Webster, Int. J. Nanomed. 6, 1553 (2011)

[10] M.P. Rayman, Proc. Nutr. Soc. 64, 527 (2005)

[11] X. Wang, K. Sun, Y. Tan, S. Wu, J. Zhang, Free Radic. Biol. Med. (2014). doi:10.1016/j.freeradbiomed.2014.04.003

[12] D. Peng, J. Zhang, Q. Liu, E.W. Taylor, J. Inorg. Biochem. 101, 1457 (2007)

[13] H. Wang, J. Zhang, H. Yu, Free Radic. Biol. Med. 42, 1524 (2007)

[14] Y. Li, X. Li, W. Zheng, C. Fan, Y. Zhang, T. Chen, J. Mater. Chem. B. 1, 6365 (2013)

[15] M.P. Ferraz, F.J. Monteiro, C.M. Manuel, J. Appl. Biomater. Biomech. 2(2), 74 (2004)

[16] L.L. Hench, J. Biomed. Mater. Res. 41, 511 (1998)

[17] H. Oonishi, Biomaterials 12(2), 171 (1991)

[18] S.Y. Zhang, J. Zhang, H.Y. Wang, H.Y. Chen, Mater. Lett. 58, 2590 (2004)

[19] J. Carmichael, W.G. De Graff, A.F. Gazdar, J.D. Minna, J.B. Mitchell, Cancer Res. 47, 943 (1987)

[20] R.N. Raghavan, N. Somanathan, T.P. Sastry, Proc. Inst. Mech. Eng. H 227, 859 (2013)

[21] J. Zhang, J. Spallholz, in Handbook of systems toxicology, ed. by D. Casciano, S.C. Sahu (John Wiley and Sons, West Sussex, 2011)

[22] A. Paraspour, S.N. Khorasani, F.H. Mohammad, Acta. Metall. Sin. (Engl. Lett.) 26, 409 (2013)

[23] S. Senel, S.J. Clure, Adv. Drug Deliv. Rev. 56, 1467 (2004)

[24] H. Kong, J. Yang, Y. Zhang, Y. Fang, K. Nishinari, G.O. Phillips, Int. J. Biol. Macromol. 65, 155 (2014)

[25] B.T. Mayers, K. Liu, D. Sunderland, Y. Xia, Chem. Mater. 15, 3852 (2003)

[26] R.S. Soumya, V.P. Vineetha, P.L. Reshma, K.G. Raghu, PLoS ONE 8, e74411 (2013)

[27] S.B. Rodan, Y. Imai, M.A. Thiede, G. Wesolowski, D. Thompson, Z. Bar-Shavit, S. Shull, K. Mann, G.A. Rodan, Cancer Res. 47, 4961 (1987)

[28] S. Panseri, C. Cunha, T. D’Alessandro, M. Sandri, G. Giavaresi, M. Marcacci, C.T. Hung, A. Tampieri, J. Nanobiotechnol. 10, 32 (2012) 